\title{
Anticoagulation-induced severe bleeding in a patient receiving bevacizumab therapy
}

\author{
Javier Munoz $\cdot$ David Hong $\cdot$ Razelle Kurzrock
}

Received: 27 September 2011/Revised: 22 November 2011/Accepted: 24 November 2011/Published online: 15 December 2011

(c) The Japanese Society of Hematology 2011

A 56-year-old female with heavily pretreated stage 4 nonsmall cell lung cancer metastatic to brain, lungs and bone who had been diagnosed with a lower extremity deep vein thrombosis and started on low molecular weight heparin in the previous week presented to the emergency department with severe bilateral flank pain, denied fever, trauma, dysuria or gastrointestinal bleeding. The patient had just finished receiving her second cycle of a clinical trial involving erlotinib, cetuximab and bevacizumab. Restaging imaging studies performed 3 days prior to presentation showed a $43 \%$ decrease in the size of her primary lung lesion, and did not show intra-abdominal metastatic disease. Physical exam revealed a chronic diffuse cetuximabinduced rash and new sacral, lumbar, and bilateral flank hematomas (Fig. 1a-c). Hemoglobin was $7.5 \mathrm{~g} / \mathrm{dL}$, decreased from $11.5 \mathrm{~g} / \mathrm{dL}$, platelets were $120 \mathrm{~K} / \mathrm{uL}$, which were at baseline, and coagulation studies, including activated partial thromboplastin time (APTT), international normalized ratio (INR), and fibrinogen, were normal. A computed tomography (CT) of the abdomen showed collections of fluid in the left paravertebral area, bilateral paraspinal regions, and adjacent to the left psoas, likely representing hematomas (Fig. 1d). The patient was transfused with packed red blood cells resulting in normalization of hemoglobin, anticoagulation was stopped, and an inferior vena cava (IVC) filter was placed. Major bleeding has been reported to affect less than $1 \%$ of patients receiving anticoagulation. Bevacizumab has been associated with venous thromboembolic events (VTE), and bleeding in patients receiving anticoagulation after a first VTE while receiving bevacizumab-based therapy, leading to temporary or permanent discontinuation of treatment. Some known toxicities of bevacizumab include hypertension, wound healing impairment, thrombosis, bleeding, and gastrointestinal perforation, which have been hypothesized to be related to the action of this monoclonal antibody against vascular endothelial growth factor (VEGF). The concomitant use of an anticoagulant and bevacizumab may have induced such massive hemorrhage in our patient, as no alternative causes were found after a thorough analysis of the bleeding event. The use of bevacizumab together with anticoagulants will inevitably rise, as bevacizumab is increasingly being used in cancer patients, who already have an inherent predisposition to VTE due to their malignancy-induced hypercoagulability state. We should, therefore, exercise caution regarding bevacizumab-induced bleeding in patients who are already receiving anticoagulation due to a previous VTE. 


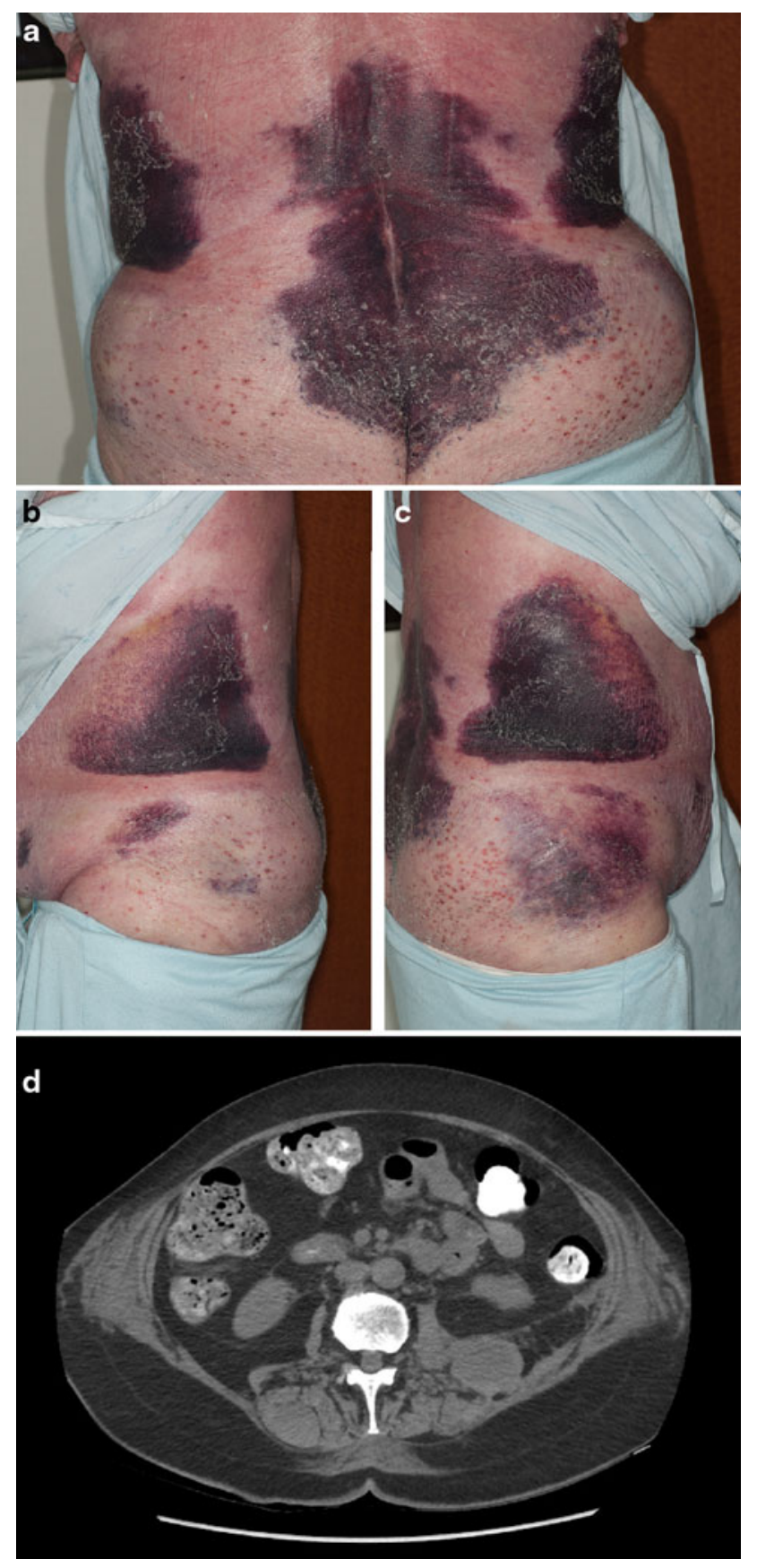

Fig. 1 a-c Physical exam revealed sacral, lumbar, and bilateral flank hematomas. d Computed tomography of the abdomen showed a collection of fluid, likely representing a hematoma, adjacent to the left psoas

\section{Conflict of interest None.}

